\title{
A Procedure for Rapid Evaluation of the Discriminative Stimulus Effects of Drugs
}

\author{
Albert J. Bertalmio, Seymore Herling, Randolph Y. Hampton, Gail Winger, \\ AND JAMES $H$. WOODS
}

\begin{abstract}
Rhesus monkeys were trained in a two-lever drug-discrimination procedure with several discrete trials per session. During training sessions, either a sham injection or a subcutaneous injection of the training drug was administered ten minutes prior to each trial. During each trial, completion of 100 consecutive responses on the lever appropriate for the animal's pharmacological condition (e.g., left for sham, right for drug) resulted in the delivery of three grams of food. Training sessions consisted of from zero to four sham trials that preceded two consecutive drug trials. The number of sham trials varied unsystematically to preclude discrimination of the drug trials on the basis of the number of preceding trials. Discriminations were established with each of the training drugs employed (codeine, methohexital, and ketamine). During dose-effect evaluations of the training drug or other drugs (test sessions), a progressively larger dose of drug was injected prior to each trial and 100 consecutive responses on either the sham- or drug-appropriate lever resulted in the delivery of food. Test sessions continued until either drug-lever responding or a marked suppression in the rate of responding occurred. Thus, a cumulative dose-effect curve for each drug was generated within a single session. Preliminary findings suggest that the pattern of cross-drug generalization generated by this cumulative-dosing procedure is similar to that obtained with procedures that evaluate only a single dose of drug per session.
\end{abstract}

Key Words: Drug discrimination; Cumulative-dose effects; Multiple-trial procedure; Rhesus monkeys

\section{INTRODUCTION}

Drug-induced discriminative control of behavior is a phenomenon of both practical and theoretical interest. In many studies of this phenomenon, an animal is trained by reinforcing a response unit following the injection of a drug and reinforcing a different response unit following a saline injection. Other drugs are then injected to determine whether they also produce the response controlled by the training drug, i.e., whether there is stimulus generalization between the training drug and the test drug. Drug generalization has been advanced as a specific assay for the classification of drugs (e.g., Barry, 1974) and as a predictor of subjective

From the Departments of Pharmacology and Psychology, University of Michigan, Ann Arbor, Michigan.

Address reprint requests to: James H. Woods, Department of Pharmacology, University of Michigan Medical School, Ann Arbor, Ml 48109.

Received January 8, 1982; revised and accepted March 9, 1982. 
effects of drugs in man (Holtzman et al., 1977; Colpaert, 1978). Procedures currently used in drug discrimination studies, however, are time consuming, particularly when compared to some in vitro assays of pharmacological activity (e.g., the isolated guinea-pig intestine: van Rossum and van den Brink, 1963).

The time required to evaluate the discriminative properties of drugs could be substantially reduced by employing a cumulative-dosing procedure to generate an entire dose-response curve during the course of a single session. Hanson et al. (1966) used such a technique to assess the relative potencies of a number of drugs administered orally to squirrel monkeys in an avoidance procedure, and Boren (1966) suggested the general utility of cumulative dosing for assessing the behavioral effects of drugs. Kelleher and Goldberg (1979) studied the behavioral effects of morphine or naloxone and the antagonism of morphine's effects by naloxone in rhesus monkeys using a cumulative-dosing technique. The approach has also been used in mice (Wenger, 1980). In addition to substantially increasing the rate of drug evaluation, these procedures produced results that agreed well with findings produced by the evaluation of single doses.

We have found that a cumulative-dose approach can be applied to the evaluation of the discriminative stimulus properties of drugs in a procedure that involves several discrete trials per session. An animal can be trained to respond on one lever during the initial trials of a session if these trials have been preceded by a sham injection, and to respond on another lever on a subsequent trial if that trial is preceded by an injection of the training drug. If an animal is trained in this manner, stimulus generalization to other drugs can be assessed by administering successively larger doses of a drug prior to each trial of a test session to determine whether the test drug produces responding similar to that produced by the training drug. With this procedure, a cumulative dose-effect curve can be obtained within a single session.

\section{MATERIALS AND METHODS}

\section{Subjects}

The subjects were four adult male rhesus monkeys (Macaca mulatta) weighing 8.0 to $9.0 \mathrm{~kg}$. Each monkey was housed individually and given a restricted amount of food (Purina Monkey Chow) in one daily feeding following the experimental session; water was freely available in the home cage. Fresh fruit was provided several times per week, and isoniazid $(40 \mathrm{mg})$ was administered daily on a sugar cube.

Each monkey $(28,639,732$, and 808$)$ had a complex experimental and drug history, including fixed-ratio responding maintained by alternate presentation of food and drug. Immediately prior to this study, three of the four animals $(28,639$, and 732$)$ had participated in another drug discrimination study. For two of the three animals, the training drug had been codeine; for the other (28), the training drug had been pentobarbital. 


\section{Apparatus}

The experimental chambers were modifications of those described by Downs and Woods (1974). Each chamber was equipped with two response levers (BRS/LVE, Beltsville, MD, model PRL-110/121-07) mounted equidistant from a food receptacle that was mounted on the center of the front wall. The levers and food receptacle were approximately $50 \mathrm{~cm}$ above the floor and were within easy reach of the monkey. The chamber was ventilated by an exhaust fan, and a speaker mounted inside the chamber provided white masking noise.

An array of colored 7-w bulbs was mounted inside the chamber at the top of the front wall. Banana-flavored food pellets ( $300 \mathrm{mg}$, Formula G, P.J. Noyes Co., Lancaster, NH) were delivered to the food receptacle by a pellet dispenser (Ralph Gerbrands Co., Arlington, MA, Model A) mounted outside the chamber. Programming, recording, and data collection were accomplished using a Texas Instruments $960 \mathrm{~A}$ computer and cumulative recorders (Ralph Gerbrands Co., Model C-3).

\section{Training Procedure}

Prior to each daily session, each monkey was removed from its home cage and placed in a primate restraining chair. Monkey and chair were then placed in the experimental chamber, and the monkey was given a subcutaneous injection of drug or a sham injection. A sham injection consisted of all of the handling involved in administering a subcutaneous injection, except that a needle was not inserted under the animal's skin. Sham injections were used instead of injecting saline to eliminate the tissue trauma that would otherwise be associated with repeated insertions of needles. Following the injection, the chamber was closed and a ten minute blackout ensued during which responses on the two levers were recorded but had no scheduled consequences. At the end of the blackout period, two blue lights were illuminated and the discrimination contingencies were then in effect.

Initially, cach press of the lever designated as correct for the session resulted in the delivery of a food pellet. The designation of "correctness" depended on the injection given prior to the session; e.g., the right lever after drug and the left lever after sham. The training drug doses were $1.0 \mathrm{mg} / \mathrm{kg}$ ketamine $(808), 3.2 \mathrm{mg} / \mathrm{kg}$ codeine $(639,732)$, or $10.0 \mathrm{mg} / \mathrm{kg}$ methohexital (28). Training doses were selected on the basis of results from preliminary experiments. The training drug for monkey 28 was switched from pentobarbital to methohexital prior to the start of this study because methohexital has a more rapid onset as a discriminative stimulus than does pentobarbital when administered subcutaneously (G. Winger and S. Herling, unpublished observations). The initial training sessions ended after 75 food deliveries or after one hour, whichever occurred first. Sessions were conducted six days per week.

For monkey 808 , the number of responses on the correct lever required to produce food was increased across sessions to 100 , with the added requirement that these responses occur in succession, without any intervening responses on the incorrect lever. For the other three monkeys $(28,639$, and 732$)$, the consecutive 
response requirement was increased initially to 20; drug discriminations were established, and drug generalizations were studied under conditions as described elsewhere (Woods et al., 1980; Herling and Woods, 1981; Winger and Herling, 1982). Subsequently, the consecutive response requirement for these three monkeys was also increased to 100 , and the final phase of training was initiated for all monkeys.

During this last phase of training, each session consisted of several discrete trials. Prior to the start of each trial, the chamber was opened and the monkey was given either a sham injection or a subcutaneous drug injection. After a ten minute blackout period, the lights were illuminated and completion of 100 consecutive responses on the lever designated as correct for the trial resulted in the delivery of $3 \mathrm{~g}$ of food, and the trial terminated. Responses on the incorrect lever reset the response requirement on the correct lever to 100 . If 100 consecutive correct responses were made before five minutes had clapsed, the remainder of the five minutes was spent in a blackout period during which responses had no scheduled consequences. If the animal failed to emit 100 consecutive responses on the appropriate lever, the trial terminated automatically after five minutes. At the end of five minutes, whether food had been delivered or not, the chamber door was opened and the monkey received the ncxt injection, ten minutes prior to the start of the next trial. Thus, the time between successive injections was constant (i.e., fifteen minutes) irrespective of the rate of responding on a particular trial.

Each training session began with from zero to four sham trials and ended with two drug trials. The number of sham trials varied unsystematically to prevent the detection of the drug trials on the basis of the number of preceding sham trials. All sham trials were preceded by sham injections, but only the first of the two drug trials was preceded by an injection of the training drug. The second drug trial was preceded by a sham injection, since drug was assumed still present from the first injection; e.g., each of the doses of the training drugs used in this study has discriminative effects in rhesus monkeys that last at least 40 minutes (unpublished observations). A discrimination was considered to be established when a monkey made a minimum of $90 \%$ correct responses on each trial for five consecutive training sessions.

\section{Test Procedure}

Test sessions were identical to training sessions except that a) on each trial of a test session, 100 consecutive responses on either the sham-appropriate or drugappropriate lever resulted in the delivery of $3 \mathrm{~g}$ of food, and b) each test session trial was preceded by the subcutaneous injection of drug, with the dose injected on each trial increasing from the first to the last trial of the session. The dose injected before each test trial was calculated to increment the total amount injected within the session by $1 / 4$ (e.g., from 0.3 to $0.56 \mathrm{mg} / \mathrm{kg}$ ) or $1 / 2$ (e.g., from 0.3 to 1.0 $\mathrm{mg} / \mathrm{kg}$ ) log-unit steps. In general, testing continued until the animal made $90 \%$ of its responses during a given trial on the drug-appropriate lever or until the rate of responding was markedly suppressed. Training and test sessions alternated. If a monkey failed to meet criterion on a training session (i.e., $90 \%$ correct responses 
on each trial of the session), further testing was postponed until the performance criterion was attained for at least two consecutive training sessions.

To assess the reliability of the cumulative-dosing procedure, the dose-effect curve for each of the training drugs was redetermined $2-3$ times in each monkcy. The first redetermination of each dose-effect curve occurred several months after the original determination of the dose-effect curve. Subsequent redeterminations were conducted at approximately one-week intervals. Training sessions and test sessions (including tests sessions with drugs not reported here) intervened between redeterminations of the training drug dose-effect curves. Finally, dose-effect curves generated by the injection of cumulative doses were compared to those obtained by the injection of single doses that were not preceded by lower doses. Single-dose determinations were obtained during sessions in which a dose of the training drug was administered prior to a single trial. In these sessions, the monkeys were removed from the chamber after one trial.

\section{Drugs}

Ethylketazocine methane sulfonate and cyclazocine (base) (Sterling-Winthrop Research Institute, Rensselaer, NY) were dissolved in sterile water to which a small amount of lactic acid was added; sodium hydroxide was used to adjust the $\mathrm{pH}$ of the solutions to above 4. Dextrorphan tartrate and levorphanol tartrate (HoffmannLa Roche, Inc., Nutley, NJ), phencyclidine hydrochloride and etorphine hydrochloride (NIDA), fentanyl citrate (Janssen Pharmaceuticals, Beerse, Belgium), ketamine hydrochloride (Warner-Lambert, Ann Arbor, MI) and codeine phosphate (S. B. Penick and Co., Lyndhurst, NJ) were dissolved in $0.9 \%$ sterile saline. Sodium pentobarbital (Ganes Chemical Works, New York, NY) was dissolved in ethanol, propylene glycol, and sterile water in a ratio of $1: 2: 7$. SKF-10,047 hydrochloride (Dr. A. E. Jacobson, NIH) and methohexital sodium (Eli Lilly and Co., Indianapolis, IN) were dissolved in sterile water. Doses refer to the forms described.

\section{RESULTS}

Discriminations were established in each monkey with either codeine, methohexital, or kctamine. Monkey 808 had not received drug discrimination training prior to this experiment and required 52 sessions to reach criterion level performance. For the monkeys with previous drug discrimination experience, 40 to 45 sessions were required to reach criterion. A criterion level training session is illustrated in Figure $1 \mathrm{~A}$ for monkey 732 trained to discriminate $3.2 \mathrm{mg} / \mathrm{kg}$ codeine from a sham injection. Ten minutes after sham injections (S), which preceded the first three trials, responses were emitted exclusively on the sham-appropriate lever. Response rates for these trials averaged 3.23 responses a second. On the two trials following the injection of $3.2 \mathrm{mg} / \mathrm{kg}$ codeine, responses were made almost exclusively on the codeine-appropriate lever. In this monkey, rates of responding following codeine averaged 2.57 responses a second. In general, few, if any, responses occurred during the blackout periods that preceded each trial (e.g., Figure 1A). When re- 


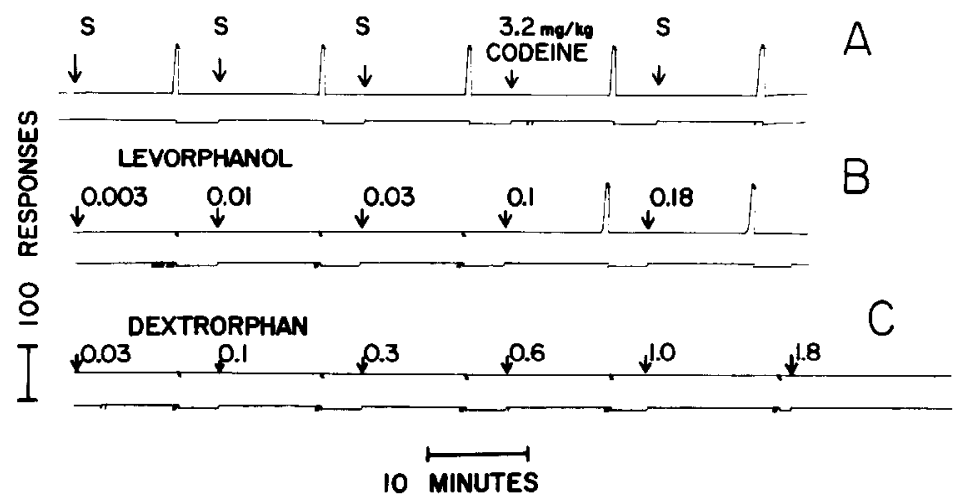

FIGURE 1. Cumulative response records from one training session (A) and two test sessions $(B, C)$ in a codeine-trained monkey (732). For each trial in record $A$, correct responses are displayed as upward steps on the upper response pen and incorrect responses are indicated as momentary deflections of the lower event pen, regardless of whether correct responses were on the drug or sham lever. Ten minutes prior to the start of a trial, a sham injection (S) or an injection of codeine $(3.2 \mathrm{mg} / \mathrm{kg}$ ) was administered. During the ten-minute pre-trial interval, the chamber was unlighted; responses were recorded, but had no scheduled consequences. Delivery of 10 food pellets $(3 \mathrm{~g})$ is marked by 10 successive downward diagonal deflections of the response pen. Upon completion of food delivery, the response pen reset and the lower event pen deflected for the remainder of the five-minute trial. During this time, the chamber was unlighted and responses had no scheduled consequences. Cumulative record B illustrates a test session in which increasing doses of levorphanol were administered. During test sessions, sham-appropriate responses are shown as deflections of the lower event pen, and codeine-appropriate responses are recorded as upward steps of the upper response pen. Numbers above each trial indicate the total dose administered up to that point in the session. Record $\mathbf{C}$ shows the results of a test session with dextrorphan.

sponses were emitted during the inter-trial intervals, these did not always occur on the lever designated as correct for the upcoming trial.

Panels $B$ and $C$ of Figure 1 show cumulative records for test sessions in which cumulative doses of levorphanol, a narcotic analgesic, and dextrorphan, the nonnarcotic dextro-isomer of levorphanol, were administered. Cumulative doses of levorphanol (panel B) up to $0.03 \mathrm{mg} / \mathrm{kg}$ produced only sham-appropriate responding. As the dose of levorphanol was increased to $0.1 \mathrm{mg} / \mathrm{kg}$ and higher, responses were made exclusively on the codeine-appropriate lever. In contrast, dextrorphan (panel C) resulted in only sham-appropriate responding even after a cumulative dose of $1.0 \mathrm{mg} / \mathrm{kg}$. The rate of responding following this dose of dextrorphan was 0.45 responses a second. A higher dose of dextrorphan, $1.8 \mathrm{mg} / \mathrm{kg}$, completely abolished responding in this monkey. Note that with both test drugs, responding on a particular trial tended to occur on only one of the two levers.

Codeine, etorphine, fentanyl, and levorphanol, each produced dose-related responding on the codeine-appropriate lever when tested in the two monkeys trained to discriminate injections of $3.2 \mathrm{mg} / \mathrm{kg}$ codeine from sham injections (Figure 2, upper panels). Dose-response curves were generally steep, with little intermediate 


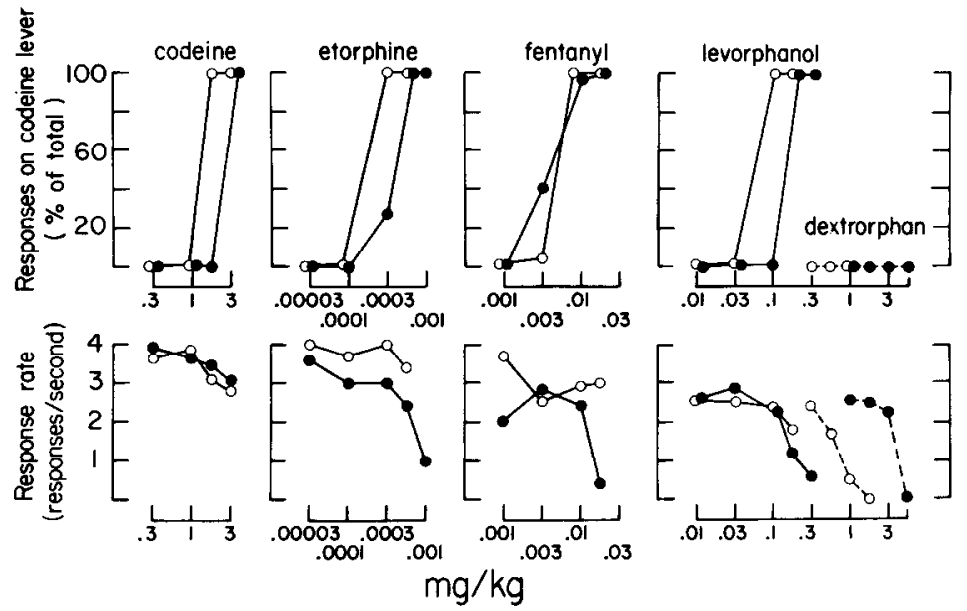

FIGURE 2. Dose-response curves for codeine, etorphine, fentanyl, levorphanol, and dextrorphan in two monkeys (639: closed circles; 732: open circles) trained to discriminate 3.2 $\mathrm{mg} / \mathrm{kg}$ codeine from sham injections. Upper panel ordinates: percentage of total responding that occurred on the codeine-appropriate lever. Lower panel ordinates: overall rate of responding. Abscissae: cumulative dose.

responding. The change from sham-appropriate to codeine-appropriate responding in these monkeys usually occurred at doses of the drugs that did not substantially affect the rate of responding (Figure 2, lower panels). In contrast, dextrorphan (Figure 2), at doses up to and including those that suppressed the rate of responding, resulted in only sham-appropriate responding in these two monkeys. Similarly, ethylketazocine $(0.001-0.01 \mathrm{mg} / \mathrm{kg})$, cyclazocine $(0.0003-0.03 \mathrm{mg} / \mathrm{kg}), \mathrm{SKF}-10,047$ $(0.003-0.56 \mathrm{mg} / \mathrm{kg})$, ketamine $(0.1-1.8 \mathrm{mg} / \mathrm{kg})$, and methohexital $(0.3-5.6 \mathrm{mg} / \mathrm{kg})$ produced exclusively sham-appropriate responding (data not shown). Each of these drugs caused dose-related decreases in the rate of responding in both monkeys.

In monkey 28 , trained to discriminate an injection of methohexital $(10.0 \mathrm{mg} / \mathrm{kg})$ from a sham injection, both methohexital and pentobarbital produced dose-related responding on the methohexital-appropriate lever, with methohexital being approximately 3 times more potent than pentobarbital. The lowest cumulative doses of methohexital and pentobarbital that resulted in $100 \%$ drug-appropriate responding were 3 and $10 \mathrm{mg} / \mathrm{kg}$, respectively. In monkey 808 , trained to discriminate an injection of ketamine $(1.0 \mathrm{mg} / \mathrm{kg})$ from a sham injection, both ketamine and phencyclidine produced dose-related drug-appropriate responding; phencyclidine was 6 times more potent than ketamine. Codeine $(0.1-3.2 \mathrm{mg} / \mathrm{kg})$ produced only shamappropriate responding in these two monkeys, even at doses that markedly reduced response rates.

When dose-effect curves for the training drugs were redetermined, differences in the lowest dose of drug that produced the maximum drug-appropriate response were small, ranging in individual monkeys from $1 / 4$ to $1 / 2$ log unit (Figure 3 , top panels). Dose-effect curves determined when drug doses were not preceded by 

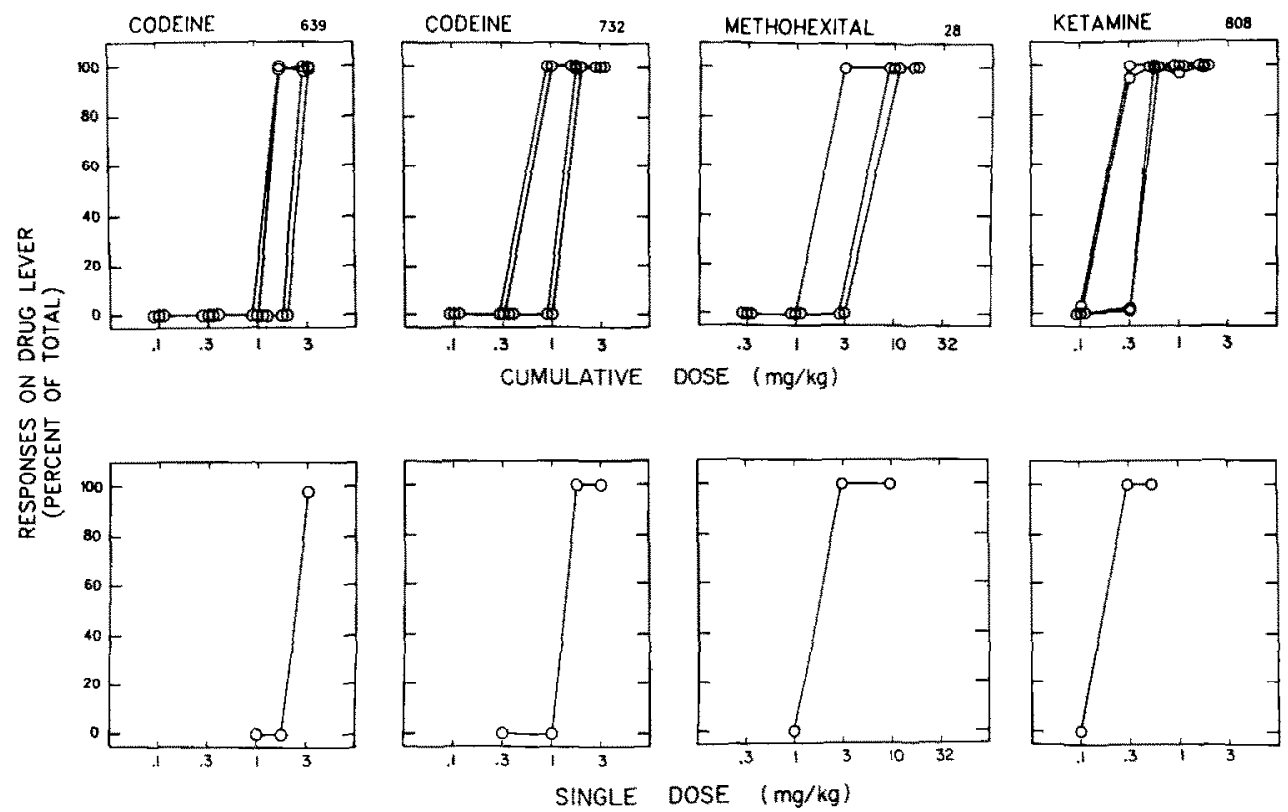

FIGURE 3. Dose-response curves for each of the training drugs determined either by cumulative dosing (top panels) or by single doses (bottom panels). Ordinates: percentage of total responding that occurred on the drug-appropriate lever. Abscissae, top panels: cumulative dose. Abscissae, bottom panels: dose administered as a single injection without preceding lower doses. Cumulative dose-effect curves were determined 3-4 times in each monkey.

lower doses (i.e., single-dose determinations; Figure 3, bottom panels) were in all respects similar to those determined by cumulative dosing (Figure 3, top panels).

\section{DISCUSSION}

The present study demonstrates that rhesus monkeys can be trained to respond on one lever following a sham injection and, within the same experimental session, to respond on a second lever following an injection of drug. Furthermore, the application of cumulative dosing during test sessions resulted in reliable and pharmacologically valid data, while increasing substantially the rate at which drugs were evaluated. In contrast to the present procedure, most commonly employed drug discrimination procedures allow for only a single dose of drug to be evaluated during a test session. The necessity for training sessions to be interpolated between test sessions reduces to two or three the number of doses of a test drug that are evaluated per week. For the data reported in the present experiment, there was approximately a three-to-six-fold increase in the rate at which test drugs were evaluated when compared to similar data generated by the use of a single-dosing procedure (e.g., Hein et al., 1981; Herling and Woods, 1981). Of course, the relative 
speed of drug evaluation realized by using a cumulative-dosing procedure rather than a single-dose procedure will depend on a number of factors including the time required to establish the discrimination, the number of points on the dose-effect curve, the number of interpolated training sessions, and the pharmacokinetics of the drugs studied.

In addition to an increase in productivity, the cumulative-dose procedure resulted in a saving in the amount of test drug used, an important consideration in the evaluation of expensive drugs or those in limited supply. On the other hand, the multiple-trial procedure led to some increase in session length, especially on test sessions, and more staff time was required while the session was in progress.

The procedure used in the present study, of course, might produce results that differ from those obtained using procedures that administer only one dose of drug prior to the session, thus limiting the generality of results obtained under a cumulative-dosing procedure. There appear, however, to be no major qualitative differences in the patterns of drug generalizations obtained in the present study and those obtained under single-dose procedures (cf. Schaefer and Holtzman, 1977; Woods et al., 1980; Herling and Woods, 1981). For example, the morphine-like compounds levorphanol, fentanyl, and etorphine, resulted in codeine-appropriate responding, whereas dextrorphan, the non-narcotic dextro-isomer of levorphanol, produced only sham-appropriate responding. Similarly, the nonmorphine-like narcotics ethylketazocine, cyclazocine, and SKF-10,047 resulted in only sham-appropriate responding in the rhesus monkeys trained to discriminate codeine from saline, as did the non-narcotics ketamine and methohexital.

The degree to which cumulative- and single-dosing procedures might differ quantitatively will depend on a number of factors (Boren, 1966). For example, a cumulative dose-response curve determined for a drug with either a very slow onset of action or with a very short half-life might underestimate the drug's potency. The cumulative dosing used in the present experiments involved observing the final effect of past drug administrations. It is important to understand how pharmacokinetic parameters might affect a dose-response curve obtained this way. It is clear that in the case of drugs with a rapid onset and long duration of effect, a cumulative dose-effect curve will accurately predict the effects obtained using single-dose techniques. Each of the training drugs used in the present experiment has a relatively rapid onset of action, i.e., the drugs produced discriminable effects within 10 minutes of their injection, and the dose-effect curves generated cumulatively resembled those obtained when only single doses were tested (Figure 3). Moreover, when the reliability of the cumulative-dosing procedure was assessed by redetermining doseresponse curves to the training drugs, the minimum dose necessary to produce drug-appropriate responding varied $1 / 4$ to $1 / 2 \log$ unit. This degree of variability is not outside the range of variability found in drug discrimination studies that test single doses (e.g., Shannon and Holtzman, 1977).

For any whole-animal assay to accurately gauge a drug's effect at a given time, the drug's time parameters must be understood. Animals trained under the multipletrial procedure described in this study are well suited to time-function measurements since they are trained to indicate the presence of a drug's discriminative 
effect during the session. To assess the onset of the discriminative effect of a drug, an animal, trained under the procedure described in this study, could be given a single injection of a drug at the beginning of a session and tested at various time points after the drug's administration (e.g., Woods et al., 1981).

In addition to increasing the rate at which data are collected, the multiple-trial cumulative-dosing approach may be helpful in addressing other pharmacological issues. For example, the rapidity of the procedure could increase the productivity of preparations in which limited life is an important consideration, e.g., intravenous, intraventricular, and intracercbral administration preparations. Furthermore, the ability to rapidly generate dose-effect curves may be useful in experiments where the major dependent variable is a shift in dose-response curves; e.g., in the study of phenomena such as drug antagonism (Kelleher and Goldberg, 1979), supersensitivity (Kelleher and Goldberg, 1979; Spealman et al., 1981), or tolerance. Since a dose-effect curve can be generated in a relatively short period of time, conclusions can be drawn about changes in the position of the curve under relatively unchanging conditions.

This research was supported by USPHS Grants DA 00254, DA 00154, and DA 02230. R. Y. Hampton was supported as a predoctoral fellow by NIGMS Grant GM 00198. The authors would like to thank Thomas R. Ward and Kari L. George for their assistance in conducting these experiments, Isabel Herling for her assistance in preparing the manuscript, and Alice M. Young and Jonathan L. Katz for their helpful comments and editorial advice. A preliminary report of this research was presented at the Satellite Meeting of the Society for Stimulus Properties of Drugs, Anaheim, CA, April 13-14, 1980 (Pharmacol. Biochem. Behav. 13:313 (1980)).

\section{REFERENCES}

Barry H III (1974) Classification of drugs according to their discriminable effects in rats. Fed Proc 33:1814-1824.

Boren JJ (1966) The study of drugs with operant techniques. In Operant Behavior: Areas of Research and Application. Ed., WK Honig. New York: Appleton-Century-Crofts, pp. 531-564.

Colpaert FC (1978) Discriminative stimulus properties of narcotic analgesic drugs. Pharmacol Biochem Behav 9:863-887.

Downs DA, Woods JH (1974) Codeine and cocainereinforced responding in rhesus monkeys: effects of dose on response rates under a fixedratio schedule. J Pharmacol Exp Ther 191:179-188.

Hanson HM, Witoslawski II, Campbell EH, Itkin AC (1966) Estimation of relative antiavoidance activity of depressant drugs in squirrel monkeys. Arch Int Pharmacodyn 161:7-16.

Hein DW, Young AM, Herling S, Woods JH (1981) Pharmacological analysis of the discriminative stimulus characteristics of ethylketazocine in the rhesus monkey. I Pharmacol Exp Ther 218:7-15.

Herling S, Woods JH (1981) Discriminative stimulus effects of etorphine in rhesus monkeys. Psychopharmacology 72:265-267.

Holtzman SG, Shannon HE, Schaefer GJ (1977) Discriminative properties of narcotic antagonists. In Discriminative Stimulus Properties of Drugs. Ed., H. Lal. New York: Plenum Press, pp. 47-72.

Kelleher RT, Goldberg SR (1979) Effects of naloxone on schedule-controlled behavior in monkeys. In Endorphins in Mental Health Research. Eds., E Usdin, WE Bunney, NS Kline. New York: Oxford University Press, pp. 461-472.

Schaefer GJ, Holtzman SG (1977) Discriminative effects of morphine in the squirrel monkey. J Pharmacol Exp Ther 201:67-75.

Shannon HE, Holtzman SG (1977) Further evaluation of the discriminative effects of morphine in the rat. I Pharmacol Exp Ther 201:55-66. 
Spealman RD, Kelleher RT, Morse WH, Goldberg SR (1981) Supersensitivity to the behavioral effects of opiate antagonists. Psychopharmacol Bull 17:54-56.

van Rossum JM, van den Brink FG (1963) Cumulative dose-response curves. I. Introduction to the technique. Arch Int Pharmacodyn 143:240-246.

Wenger GR (1980) Cumulative dose-response curves in behavioral pharmacology. Pharmacol Biochem Behav 13:647-651.

Winger G, Herling S (1982) Discriminative stimulus effects of pentobarbital in rhesus monkeys: Tests of stimulus generalization and duration of action. Psychopharmacology (to appear).

Woods JH, Herling S, Valentino RJ, Hein DW, Coale EC (1980) Narcotic drug discriminations by rhesus monkeys and pigeons. In Problems of Drug Dependence, 1979, NIDA Research Monograph 27. Fd., IS Harris. Washington: U.S. Govt Printing Office, pp. 128-134.

Woods JH, Herling S, Young AM (1981) Comparison of discriminative and reinforcing stimulus characteristics of morphine-like opioids and two met-enkephalin analogues. Neuropeptides 1:409-419. 